\title{
Pulsations of massive ZZ Ceti stars with carbon/oxygen and oxygen/neon cores
}

\author{
A. H. Córsico ${ }^{1,2, \star}$, E. García-Berro ${ }^{3,4}$, L. G. Althaus ${ }^{1,2,3, \star \star}$, and J. Isern ${ }^{3,5}$ \\ ${ }^{1}$ Facultad de Ciencias Astronómicas y Geofísicas, Universidad Nacional de La Plata, Paseo del Bosque, s/n, (1900) La Plata, \\ Argentina \\ e-mail: [acorsico;althaus]@fcaglp.unlp.edu.ar \\ 2 Instituto de Astrofísica La Plata, IALP, CONICET, Argentina \\ 3 Departament de Física Aplicada, Universitat Politècnica de Catalunya, Av. del Canal Olímpic, s/n, 08860 Castelldefels, Spain \\ e-mail: garcia@fa.upc.es; isern@ieec.fcr.es \\ ${ }^{4}$ Institut d'Estudis Espacials de Catalunya, Ed. Nexus, c/Gran Capità 2, 08034 Barcelona, Spain \\ 5 Institut de Ciències de l'Espai (CSIC)
}

Received 10 March 2004 / Accepted 16 July 2004

\begin{abstract}
We explore the adiabatic pulsational properties of massive white dwarf stars with hydrogen-rich envelopes and oxygen/neon and carbon/oxygen cores. To this end, we compute the cooling of massive white dwarf models for both core compositions taking into account the evolutionary history of the progenitor stars and the chemical evolution caused by timedependent element diffusion. In particular, for the oxygen/neon models we adopt the chemical profile resulting from repeated carbon-burning shell flashes expected in very massive white dwarf progenitors. For carbon/oxygen white dwarfs we consider the chemical profiles resulting from phase separation upon crystallization. For both compositions we also take into account the effects of crystallization on the oscillation eigenmodes. We find that the pulsational properties of oxygen/neon white dwarfs are noticeably different from those made of carbon/oxygen, thus making asteroseismological techniques a promising way to distinguish between the two types of stars and, hence, to obtain valuable information about their progenitors.
\end{abstract}

Key words. stars: evolution - stars: white dwarfs - stars: oscillations

\section{Introduction}

White dwarfs are the most common end-product of stellar evolution. Most of the presently observed white dwarfs are postAsymptotic Giant Branch (AGB) remnants, the core of which consists of the main ashes of helium burning, basically a mixture of carbon and oxygen. However, there is growing theoretical evidence suggesting that white dwarfs with masses larger than $\sim 1.05 M_{\odot}$ could have cores composed primarily of oxygen and neon (García-Berro \& Iben 1994; Ritossa et al. 1996; D'Antona \& Mazzitelli 1996; García-Berro et al. 1997; Iben et al. 1997). In particular, García-Berro et al. (1997) found that when the core mass of the $9 M_{\odot}$ white dwarf progenitor exceeds $\sim 1.05 M_{\odot}$, and before the star reaches the thermally pulsing phase at the AGB tip, carbon is ignited offcenter in semidegenerate conditions. As a result of repeated carbon-burning shell flashes that eventually give rise to carbon exhaustion in the degenerate core, it is found that at the end of the carbon burning phase the star would be left with

^ Member of the Carrera del Investigador Científico y Tecnológico, CONICET, Argentina.

$\star \star$ Member of the Carrera del Investigador Científico y Tecnológico, CONICET, Argentina. an oxygen/neon core with trace amounts of carbon and other heavier chemical species. After mass-loss episodes, the progenitor remnant is expected to evolve into the central star of a planetary nebula and ultimately into a white dwarf with an oxygen/neon core of mass $\sim 1.05 M_{\odot}$. A possible observational counterpart of these massive white dwarfs could be the single massive white dwarf LHS 4033, which has a mass of $\sim 1.32 M_{\odot}$ (Dahn et al. 2004). Other possible massive white dwarfs hosting oxygen/neon cores could be the magnetic white dwarf PG 1658+441 (Schmidt et al. 1992; Dupuis et al. 2003) - with a mass of $\simeq 1.31 M_{\odot}$ - and the highly magnetic white dwarf RE J0317-853 (Barstow et al. 1995; Ferrario et al. 1997), which has a mass of $\sim 1.35 M_{\odot}$. It should be noted, however, that there are alternative evolutionary channels that could eventually lead to massive white dwarfs with carbon/oxygen cores. These scenarios involve the merging of two otherwise lightweight ordinary white dwarfs. It has been shown (Segretain et al. 1997; Guerrero et al. 2004) that in this case nuclear reactions are not able to modify greatly the composition of the core of the remnant.

Many white dwarfs exhibit multiperiodic luminosity variations caused by gravity-modes of low harmonic degree $(\ell \leq 2)$ 
and with periods ranging from roughly $100 \mathrm{~s}$ to approximately $1200 \mathrm{~s}$. Over the years, the study of the pulsational patterns of variable white dwarfs through asteroseismological techniques has become a very powerful tool for probing the inner regions that would be otherwise unaccessible to direct observations. In particular, for hydrogen-rich variable DA white dwarfs - also known as ZZ Ceti stars - such a technique has proved to be very successful in providing independent valuable constraints to their fundamental properties, such as their core composition, the outer layer chemical stratification or the stellar mass - see, for instance, Pfeiffer et al. (1996), and Bradley (1998, 2001). Very recently, increasing attention has been paid to the study of the asteroseismological properties of massive ZZ Ceti stars, since it opens the interesting possibility of probing the physical mechanisms operating in their very dense interiors and, particularly, to obtain useful information about the crystallization process that occurs in their cores (Montgomery \& Winget 1999). This has been motivated by the discovery of pulsations in the star BPM 37093 (Kanaan et al. 1992), a massive ZZ Ceti star which has a stellar mass of $\sim 1.05 M_{\odot}$ and an effective temperature $T_{\text {eff }} \simeq 11800 \mathrm{~K}$, and that, therefore, should have a sizeable crystallized core (Winget et al. 1997). It is interesting to note at this point that this mass is very close to the theoretical lower limit $\left(\sim 1.05 M_{\odot}\right)$ for an oxygen/neon white dwarf to be formed (Salaris et al. 1997; Gil-Pons et al. 2003). Indeed, Gil-Pons et al. (2003) find that for carbon burning to take place in the corresponding progenitor, its mass on the main sequence should be $\sim 8.1 M_{\odot}$, leaving a massive oxygen/neon white dwarf of $\sim 1.05 M_{\odot}$. Hence, BPM 37093 could be either a carbon/oxygen or an oxygen/neon white dwarf, depending on the precise value of its mass.

We do not intend to perform a detailed modelling of the pulsational characteristics of BPM 37093, since we consider it to be beyond the scope of the paper. Instead, the aim of the present paper is to assess the adiabatic pulsational properties of massive white dwarfs with carbon/oxygen (CO) and oxygen/neon $(\mathrm{ONe})$ cores. More specifically, our main goal is to explore the possibility of using white dwarf asteroseismology to distinguish between the two types of star. To this end, we compute the cooling of massive white dwarf models for both core compositions taking into account the evolutionary history of their progenitors and the chemical evolution caused by timedependent element diffusion during the evolution. The paper is organized as follows. In Sect. 2 we describe the main characteristics of our CO and ONe model white dwarfs. Special emphasis is given to the formation of the core chemical profile during the pre-white dwarf evolutionary stages. The pulsational predictions for $t$ both types of white dwarf sequences are described in Sect. 3. Finally, in Sect. 4 we briefly summarize our main findings and draw our conclusions.

\section{Input physics and evolutionary models}

In this work we compute the evolution and the pulsational properties of massive white dwarfs with ONe cores. In pursuing this goal we adopt the chemical profiles obtained by García-Berro et al. (1997) - see Sect. 2.2 for a detailed discussion. Additionally, since a major aim of our work is to compare the theoretical pulsational spectra of our ONe white dwarf models with those of their CO analogs, we also compute the evolution of a white dwarf with a $\mathrm{CO}$ core of similar mass. The most relevant results of this calculation are presented in Sect. 2.1 below. An important aspect of the present study common to both sequences concerns the evolution of the chemical abundance distribution caused by diffusion once the white dwarfs are formed. This is particularly relevant for the study of the pulsational properties of white dwarfs. Indeed, element diffusion turns out to be a key ingredient as far as mode trapping in pulsating stratified white dwarfs is concerned (Córsico et al. 2001, 2002). We have used a time-dependent treatment for multicomponent gases (Burgers 1969) which considers gravitational settling and chemical and thermal diffusion for ${ }^{1} \mathrm{H}$, ${ }^{3} \mathrm{He},{ }^{4} \mathrm{He},{ }^{12} \mathrm{C}$, and ${ }^{16} \mathrm{O}$ species, the dominant constituents outside the core of our white dwarf models - see Althaus et al. $(2001 \mathrm{a}, \mathrm{b})$ for additional details. In this way, the trace element approximation usually invoked in most ZZ Ceti studies is avoided.

\subsection{Carbon/oxygen white dwarf models}

Our massive white dwarf models with a CO core have been computed with the evolutionary code described at length in Althaus et al. (2003). The code is based on an up-to-date and detailed physical description. Briefly, the code uses OPAL radiative opacities for arbitrary metallicity from Iglesias \& Rogers (1996) and from Alexander \& Ferguson (1994) for the low-temperature regime. The equation of state for the lowdensity regime comprises partial ionization for hydrogen and helium compositions, radiation pressure and ionic contributions. For the high-density regime, partially degenerate electrons and Coulomb interactions are also considered. Under degenerate conditions we use an updated version of the equation of state of Magni \& Mazzitelli (1979). Neutrino emission rates and high-density conductive opacities are taken from the work of Itoh and collaborators - see Althaus et al. (2002). A nuclear network of 34 thermonuclear reaction rates and 16 isotopes has been considered to describe hydrogen (proton-proton chains and CNO bi-cycle) and helium burning. Nuclear reaction rates were taken from Caughlan \& Fowler (1988) except for the ${ }^{12} \mathrm{C}(\alpha, \gamma){ }^{16} \mathrm{O}$ reaction rate, for which we adopted that of Angulo et al. (1999).

The treatment of the change of the abundances during the pre-white dwarf evolution is an important aspect. In particular, our code uses a time-dependent scheme for the simultaneous treatment of chemical changes caused by nuclear burning and convective, salt finger and overshoot mixing, which are described as diffusion processes (Althaus et al. 2003). We have used the Schwarzschild criterion for convective stability. However, the occurrence of additional mixing beyond what is predicted by this criterion is suggested by both theoretical and observational evidence. In particular, extra mixing episodes (particularly mechanical overshooting and/or semiconvection) taking place beyond the formally convective boundary towards the end of central helium burning have a large influence on the carbon and oxygen distribution in the core of white 
dwarfs - see Straniero et al. (2003) for a recent discussion. Such mixing episodes leave strong signatures on the theoretical period spectrum of massive ZZ Ceti stars (Althaus et al. 2003). Therefore, we have allowed for some mechanical overshooting by following the formalism of Herwig (2000). Our mixing scheme allows for a self-consistent treatment of diffusive overshooting in the presence of nuclear burning. In particular, we have considered exponentially decaying diffusive overshooting above and below any convective region, including the convective core (main sequence and central helium burning phases), the external convective envelope and the short-lived helium-flash convection zone which develops during the thermal pulses. Finally, convection is treated according to the mixing length theory for fluids with composition gradients (Grossman \& Taam 1996) that applies in the convective, semiconvective, and salt finger instability regimes. The mass of the resulting CO white dwarf is $\approx 0.94 M_{\odot}$. However, in oder to perform a direct comparison with the ONe white dwarf model previously mentioned we have artificially scaled the stellar mass of the $\mathrm{CO}$ model to $1.06 M_{\odot}$ - approximately the mass of BPM 37093 - but taking into account the core chemical distribution expected for a progenitor star with a stellar mass correspondingly larger.

Crystallization in the core of our ZZ Ceti models and its effects on their pulsational pattern constitute an central point of this paper. Crystallization sets in when $\Gamma \equiv Z^{2} e^{2} / \bar{r} k_{\mathrm{B}} T=180$, where $\bar{r}$ is the radius of the Wigner-Seitz sphere. In addition, the chemical redistribution in the fluid above the solid core induced by phase separation upon crystallization (García-Berro et al. 1988; Segretain et al. 1994) has been taken into account by adopting the phase diagram of Segretain \& Chabrier (1993) and following the procedure described in Salaris et al. (1997) see, also, Montgomery et al. (1999). Nevertheless, for the sake of completeness we have also computed a cooling sequence in which phase separation has been neglected.

In our evolutionary sequence, the $\mathrm{CO}$ white dwarf model begins to crystallize at $T_{\text {eff }} \approx 16600 \mathrm{~K}$. In Fig. 1 we show the chemical profile of a CO white dwarf model at the ZZ Ceti stage, in which the chemical redistribution due to phase separation has been neglected. The effective temperature of the model is $T_{\text {eff }} \approx 11800 \mathrm{~K}$ and their its mass fraction amounts to 0.63 (the crystallized core is shown as a gray zone). Note the presence of a pronounced step at $\log \left(1-M_{\mathrm{r}} / M_{*}\right) \approx-0.7$ in the oxygen profile. This feature, which reflects the occurrence of overshoot episodes prior to the formation of the white dwarf, leaves strong imprints on the theoretical period spectrum (Althaus et al. 2003). Note also that the external chemical interfaces, including the He-core transition, are very smooth as consequence of the element diffusion processes acting during the evolution.

Figure 2 displays the chemical profiles of a CO ZZ Ceti model in which chemical redistribution due to phase separation has been considered at $T_{\text {eff }} \approx 11800 \mathrm{~K}$. In this case the crystallized mass fraction amounts to 0.55 , a slightly smaller value than that of the case in which phase separation was neglected (Fig. 1). This is due to the larger abundance of carbon in the overlying fluid layers resulting from the oxygen enhancement in the crystallized core of the model. Note that,

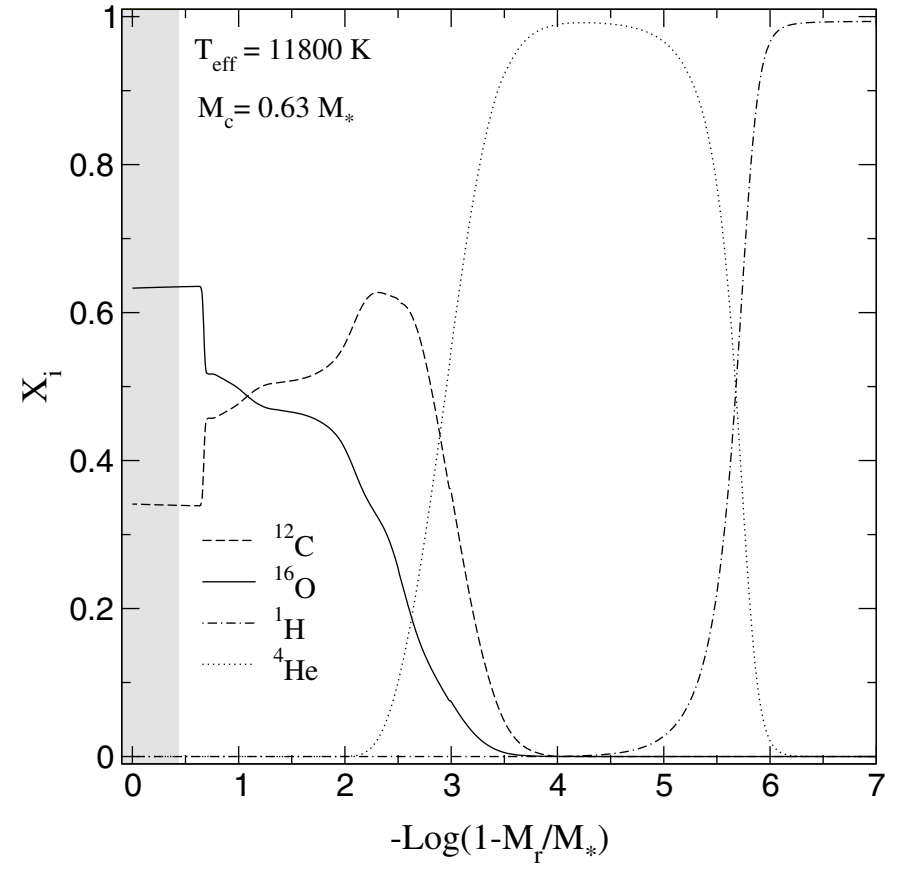

Fig. 1. The internal chemical profile corresponding to a $1.06 M_{\odot}$ $\mathrm{CO}$ model in which chemical rehomogenization induced by crystallization has been neglected. The gray area marks the domain of crystallization. $M_{\mathrm{c}}$ means the crystallized mass of the model.

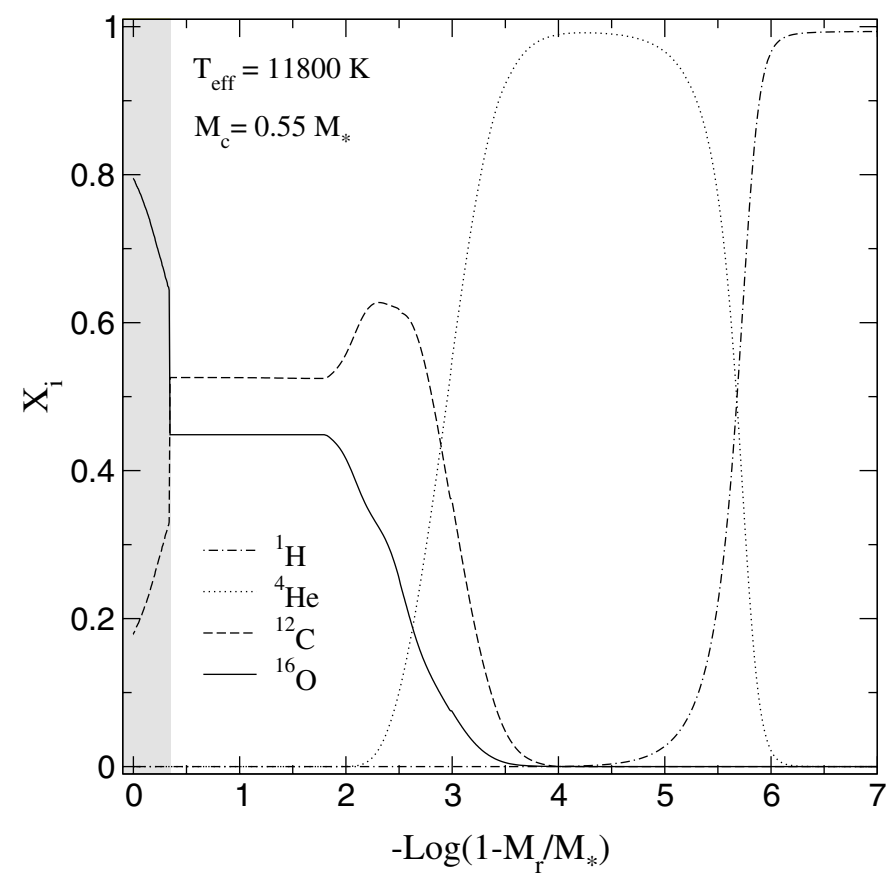

Fig. 2. Same as Fig. 1, but in the case in which chemical rehomogenization induced by crystallization has been taken into account.

as a consequence of the mixing resulting from phase separation, the overshoot-induced step in the innermost oxygen profile has been completely wiped out. Consequently, the theoretical period spectrum for this model should have much fewer strong features when compared with that of the case in which chemical rehomogenization has been neglected. This feature will be discussed in depth in Sect. 3 . 


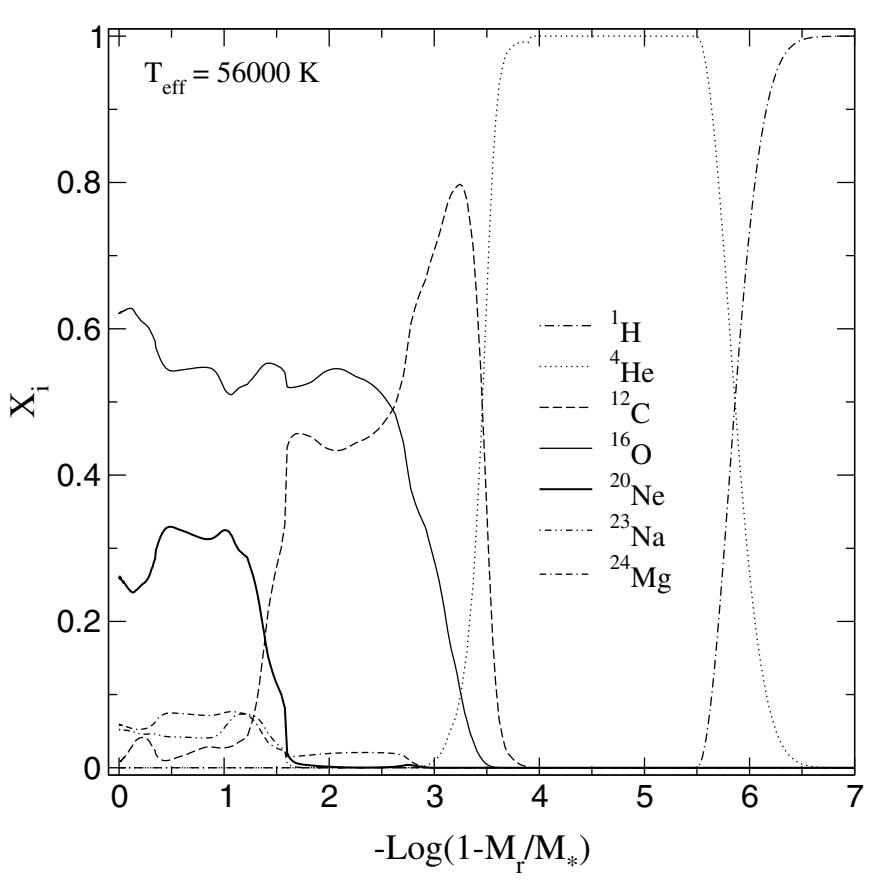

Fig. 3. The internal chemical profile corresponding to our initial $\mathrm{ONe}$ white dwarf model in terms of the outer mass fraction.

\subsection{Oxygen/neon white dwarf models}

The evolutionary stages - from the zero-age main sequence, through helium burning and off-center carbon ignition and burning in partially degenerate conditions up to the thermally pulsing AGB - leading to the formation of ONe white dwarfs, as well as the input physics employed in the calculations are described in García-Berro et al. (1997) and references therein. Here, we restrict ourselves to summarizing the main characteristics of the models and, particularly, their internal composition. When the carbon burning phase is finished, the star is left with a $\sim 1.06 M_{\odot}$ core primarily composed of ${ }^{16} \mathrm{O}$ and ${ }^{20} \mathrm{Ne}$, with trace amounts of ${ }^{12} \mathrm{C},{ }^{23} \mathrm{Na}$ and ${ }^{24} \mathrm{Mg}$. Surrounding the core there is a buffer composed mainly of a mixture of ${ }^{12} \mathrm{C}$ and ${ }^{16} \mathrm{O}$ and, on top of it, the overlying hydrogen- and heliumrich envelope layer. The initial chemical profile of the envelope - which, after diffusion, turns into a pure hydrogen layer - is shown in Fig. 3. We have obtained an initial configuration for our ONe white dwarf model by simply scaling the internal chemical profiles built up during the pre-white dwarf evolution to the structure of the massive, hot $\mathrm{CO}$ white dwarf model considered previously. In this way we obtain a good starting configuration with a mass close to that of BPM 37093 and the appropriate chemical profile. Because we are interested in the comparison of the pulsational properties of highly-evolved $\mathrm{CO}$ and $\mathrm{ONe}$ ZZ Ceti models with the same stellar mass, this procedure is adequate for our purposes.

In our evolutionary sequence, the ONe white dwarf model begins to crystallize at $T_{\text {eff }} \approx 20700 \mathrm{~K}$, long before reaching the ZZ Ceti instability strip. Figure 4 shows the resulting chemical structure when the model has reached the ZZ Ceti instability strip. At this point of the evolution, the crystallized mass fraction amounts to $\simeq 90 \%$. Two important points deserve additional comment. First, as is the case for $\mathrm{CO}$ white dwarfs,

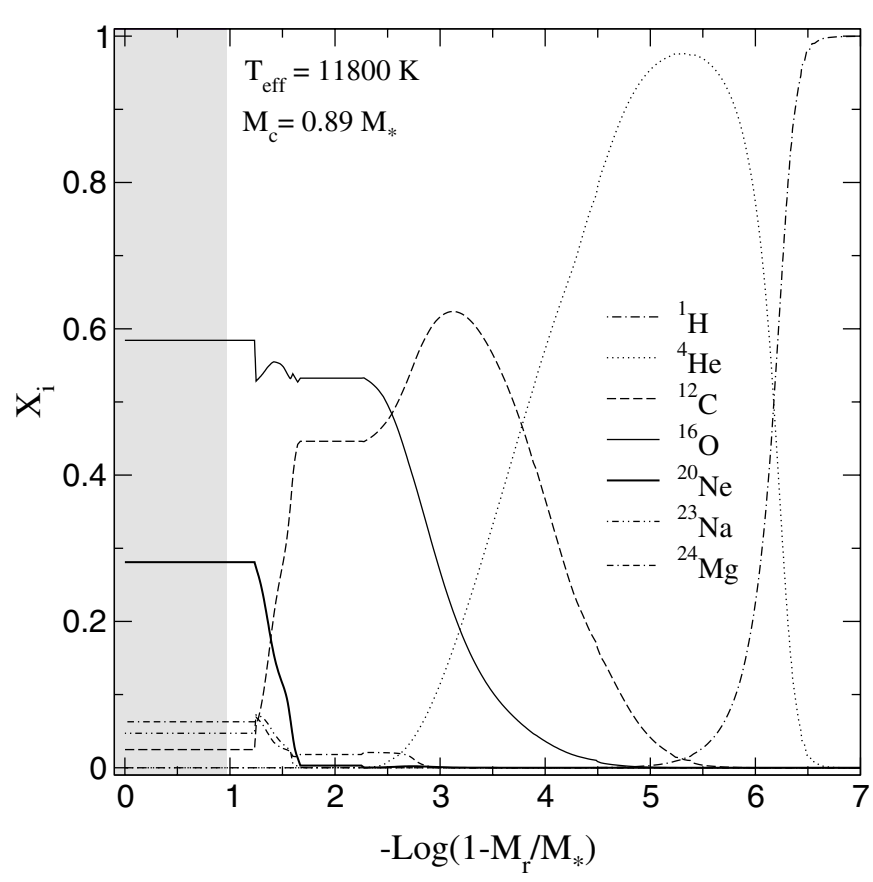

Fig. 4. Same as Fig. 3, but for an ONe white dwarf model in the ZZ Ceti instability strip. The crystallized region is displayed as a gray area.

the effects of chemical diffusion in the external chemical abundance distribution are noteworthy. Indeed, the outer chemical interfaces are markedly smoothed out by diffusion. Second, a rehomogenization process induced by Rayleigh-Taylor instabilities has led to a plateau in the innermost chemical profile by the time the ZZ Ceti stage is reached. Note that as a result, a strong step in the chemical profile at $\log \left(1-M_{\mathrm{r}} / M_{*}\right) \approx-1.25$ has arisen after rehomogenization, a feature expected to be the most important ingredient in determining the structure of the period pattern of the model. It is also important to mention here that $\mathrm{ONe}$ white dwarf models do not experience a significant chemical redistribution due to phase separation because the charge ratio of $\mathrm{Ne}$ to $\mathrm{O}$ is much smaller than that of $\mathrm{O}$ and $\mathrm{C}$ and, hence, the phase diagrams of Segretain \& Chabrier (1993) do not predict a sizeable enrichment in Ne in the solid phase. Moreover, the evolutionary calculations of Ritossa et al. (1996), García-Berro et al. (1997) and Iben et al. (1997) predict that after the carbon burning phase and when the thermally pulsing phase ensues there is a non-negligible fraction of unburnt carbon in the oxygen/neon core. The mass fraction of unburnt carbon in the ONe core can be as high as $X_{\mathrm{C}} \simeq 0.01$ - see Fig. 3. The same happens when a merger of two light-weight white dwarfs is involved (Guerrero et al. 2004). Hence, the calculation of the effects of Ne sedimentation upon crystallization would require a ternary phase diagram (Segretain 1996) which, at present, is not yet well known and, moreover, is beyond the scope of this paper.

\section{Pulsational calculations}

We have carried out an adiabatic pulsational analysis of our white dwarf models with the help of the same Newton-Raphson pulsational code employed in Córsico et al. (2001; 2002) and 
described in detail in Córsico (2003). This code is coupled to the evolutionary code previously described. However, for the purposes of this work, we have made appropriate modifications to the pulsational code in order to handle properly the effects of crystallization on the oscillation eigenmodes. Briefly, the boundary conditions at the stellar center (when crystallization has not yet set in) and surface are those given by Osaki $\&$ Hansen (1973). However, when the core of the white dwarf undergoes crystallization we switch the fluid internal boundary conditions to the so-called "hard sphere" boundary conditions (Montgomery \& Winget 1999). Within this approximation, there is an inhibition on the propagation of the nonradial eigenfunctions in the crystallized region of the core. Following previous studies of white dwarf pulsations, the normalization condition adopted is $\xi_{\mathrm{r}} / r=1$ at the stellar surface, where $\xi_{\mathrm{r}}$ the radial component of the displacement. The oscillation kinetic energy $\left(E_{\text {kin }}\right)$ is computed according to Eq. (1) of Córsico et al. (2002), whereas the weight function is computed as in Kawaler et al. (1985). Finally, we derive the asymptotic spacing of periods $\left(\Delta P_{\ell}\right)$ as in Tassoul et al. (1990):

$\Delta P_{\ell}=\frac{2 \pi^{2}}{\sqrt{\ell(\ell+1)}} \frac{1}{\int_{r_{1}}^{r_{2}} \frac{N}{r} \mathrm{~d} r}$

where $N$ is the Brunt-Väisälä frequency, and $r_{1}$ and $r_{2}$ are, respectively, the radii of the inner and outer boundaries of the propagation region of the modes. Hence, when the core is already partially crystallized $r_{1}$ coincides with the radius of the crystallization front, $r_{1}=r\left(M_{\mathrm{c}}\right)$. It is important to realize that the turning point $r_{1}$ is no longer a fixed value but, instead, it is a function of the crystallized mass $M_{\mathrm{c}}$ and hence of the temperature of the (nearly) isothermal core. As a result, as the white dwarf cools down, the internal boundary at $r_{1}$ moves outward, so the integral $\int_{r_{1}}^{r_{2}} N \mathrm{~d} r / r$ decreases, and consequently the period spacing increases and so do the periods themselves - see Figs. 7 and 8 of Montgomery \& Winget (1999).

\subsection{Brunt-Väisälä frequency}

The Brunt-Väisälä frequency is computed according to the procedure of Brassard et al. (1991). This numerical treatment takes explicit account of the contribution to $N$ from any change in composition in the white dwarf models by means of the Ledoux term $B$. This is an important aspect in connection with the phenomenon of mode trapping and mode confining (Brassard et al. 1992; Córsico et al. 2002). Specifically, the Brunt-Väisälä frequency is given by:

$N^{2}=\frac{g^{2} \rho}{p} \frac{\chi_{\mathrm{T}}}{\chi_{\rho}}\left(\nabla_{\mathrm{ad}}-\nabla+B\right)$.

The Ledoux term $B$, for the case of a multicomponent plasma, is given by

$B=-\frac{1}{\chi_{\mathrm{T}}} \sum_{\mathrm{i}=1}^{M-1} \chi_{X_{\mathrm{i}}} \frac{\mathrm{d} \ln X_{\mathrm{i}}}{\mathrm{d} \ln p}$

Here, $p$ is the pressure, $\chi_{\mathrm{T}}\left(\chi_{\rho}\right)$ denotes the partial logarithmic derivative of the pressure with respect to $T(\rho), \nabla$ and $\nabla_{\mathrm{ad}}$ are

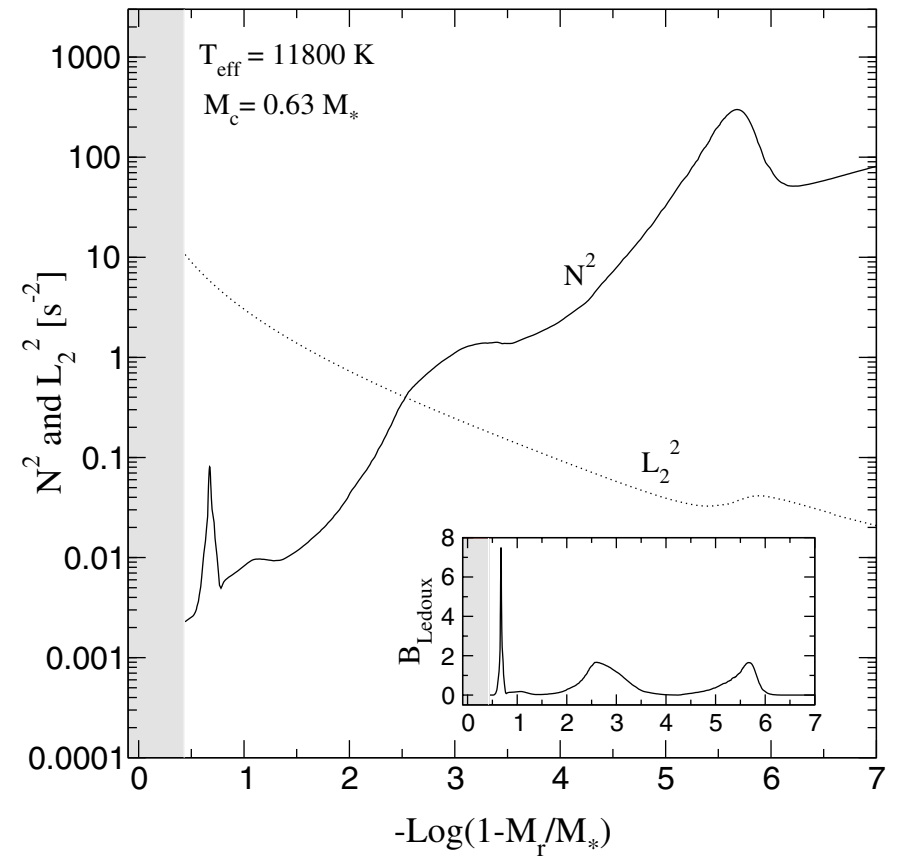

Fig. 5. The squared Brunt-Väisälä frequency $\left(N^{2}\right)$ - solid line - in terms of the outer mass fraction, corresponding to the same $\mathrm{CO}$ white dwarf model shown in Fig. 1. Inset: the Ledoux term $B$. For the sake of completeness, we also show using a dotted line the acoustic (Lamb) frequency corresponding to $\ell=2$. The gray area marks the domain of the crystallized core. Here, because of the hard-sphere approximation, the detailed shape of the Brunt-Väisälä frequency is irrelevant to pulsations and hence it is not shown.

the actual and adiabatic temperature gradients, respectively, $X_{\mathrm{i}}$ is the abundance by mass of the chemical species $i$, and

$\chi_{X_{\mathrm{i}}}=\left(\frac{\partial \ln p}{\partial \ln X_{\mathrm{i}}}\right)_{\rho, T,\left\{X_{\mathrm{j} \neq \mathrm{i}}\right\}}$.

We begin by examining Fig. 5, where the square of the Brunt-Väisälä frequency corresponding to the same $\mathrm{CO}$ white dwarf model displayed in Fig. 1 is shown. It is worth recalling that in this model phase separation upon crystallization was disregarded. Note also that in our models the shape of the outer chemical interfaces is assessed using a time-dependent element diffusion scheme. Consequently, and not surprisingly, our calculations predict the presence of very smooth bumps in the profile of $N^{2}$ in the external layers of the model (Córsico et al. 2002). Hence, and contrary to what it is usually found when the so-called "trace element approximation" is used, in our calculations these features play a minor role in the mode trapping and confining characteristics of the theoretical pulsational spectrum. Instead, it is the chemical structure of the core that determines the structure of the period spectrum, as already shown in Althaus et al. (2003). Specifically, the pulsation characteristics of this model are fixed by the presence of the pronounced peak in $N$ at $\log \left(1-M_{\mathrm{r}} / M_{*}\right) \approx-0.7$, a feature directly related to the overshoot-induced step in the chemical profile (see Fig. 1).

When phase separation upon crystallization is taken into account for the $\mathrm{CO}$ white dwarf models, chemical redistribution in the overlying fluid layers is expected to take place 


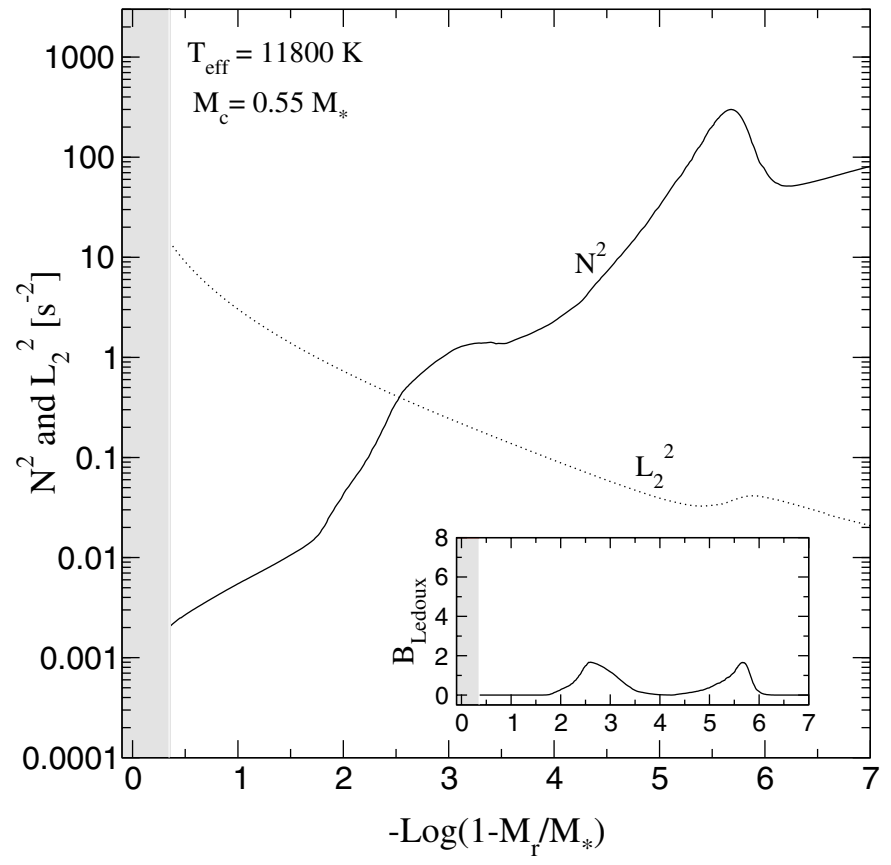

Fig. 6. Same as in Fig. 5, but for the CO white dwarf model analyzed in Fig. 2.

as a consequence of the $\mathrm{O}$ enhancement in the solid phase. Consequently, the chemical abundances (an their distribution throughout the fluid layer) of both $\mathrm{C}$ and $\mathrm{O}$ are expected to be significantly different from those of the previous model. The Brunt-Väisälä frequency corresponding to a CO white dwarf model in which the chemical profile has been computed consistenly with the predictions of chemical rehomogenization (Fig. 2) is shown in Fig. 6. Note that at this stage the CO white dwarf models have a significant percentage of its interior crystallized $(55 \%)$, in such a way that the chemical step produced by core overshooting during the pre-white dwarf evolution has been wiped out by the ongoing chemical redistribution. Because the external chemical transition regions have been smoothed by diffusion, the shape of the Brunt-Väisälä frequency has no any abrupt feature capable of strongly pertubating the period spectrum of the model. Hence, the period spacing distribution should be more or less uniform, at least in the asymptotic limit of large overtones (long periods).

Now we examine the Brunt-Väisälä frequency characterizing a typical ONe white dwarf model at the ZZ Ceti stage. In Fig. 7 we plot the profile of $N^{2}$ for the same model analyzed in Fig. 4. Clearly, the dominant feature is the one located at $-2 \lesssim \log \left(1-M_{\mathrm{r}} / M_{*}\right) \lesssim-1$ (see the Ledoux term $B$ in the inset of the figure). As we shall see in the next section, this feature is responsible for strong non-uniformities in the period spectrum of this model.

\subsection{Pulsational spectrum}

We have computed adiabatic, nonradial, spheroidal $g$ (gravity)modes with $\ell=2$ covering the period range of pulsations observed in ZZ Ceti stars. We have not considered torsional modes, since these modes, characterized by very short periods

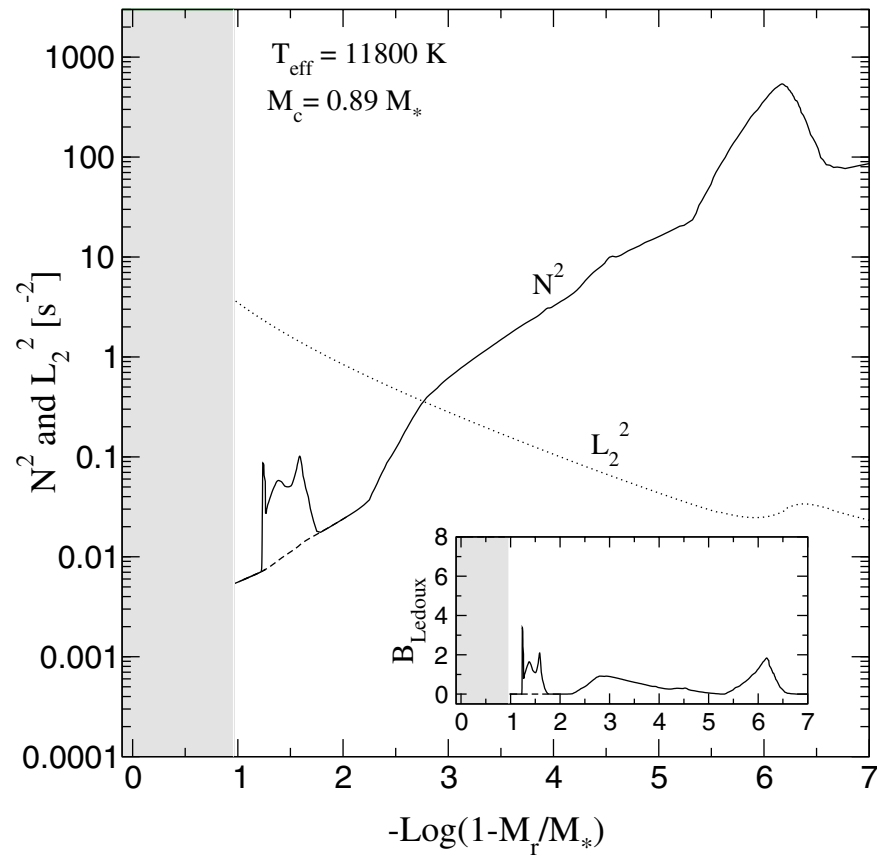

Fig. 7. Same as Fig. 6, but for the ONe white dwarf model analyzed in Fig. 4. Dashed lines correspond to the case in which the Ledoux term $B$ is neglected in the region $-2 \lesssim \log \left(1-M_{\mathrm{r}} / M_{*}\right) \lesssim-1$ (see Sect. 3.2 for additional details).

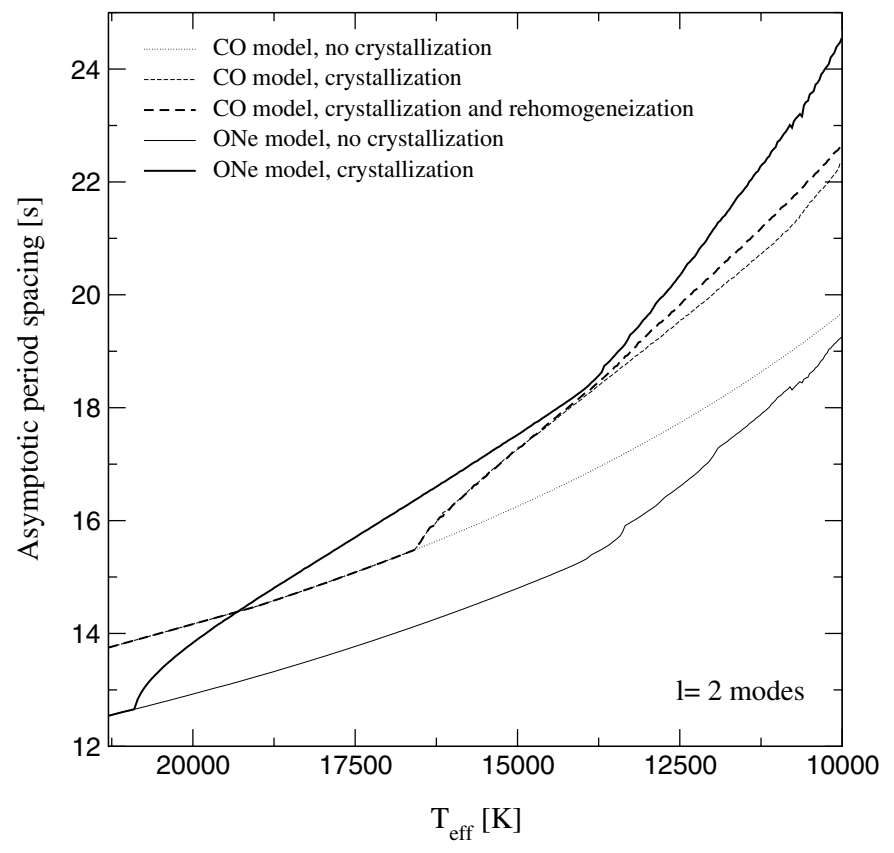

Fig. 8. Asymptotic period spacing, $\Delta P_{(\ell=2)}$, as a function of the effective temperature, for the $\mathrm{CO}$ and $\mathrm{ONe}$ evolutionary cooling sequences (thick and thin curves, respectively). See text for details.

- of up to 20 s (Montgomery \& Winget 1999) - have not been detected in ZZ Ceti stars so far.

We begin by examining Fig. 8, where the asymptotic period spacing as a function of the effective temperature is shown for the two $\mathrm{CO}$ and the $\mathrm{ONe}$ white dwarf models previously discussed, and for two additional models in which crystallization has been completely disregarded. These two additional 

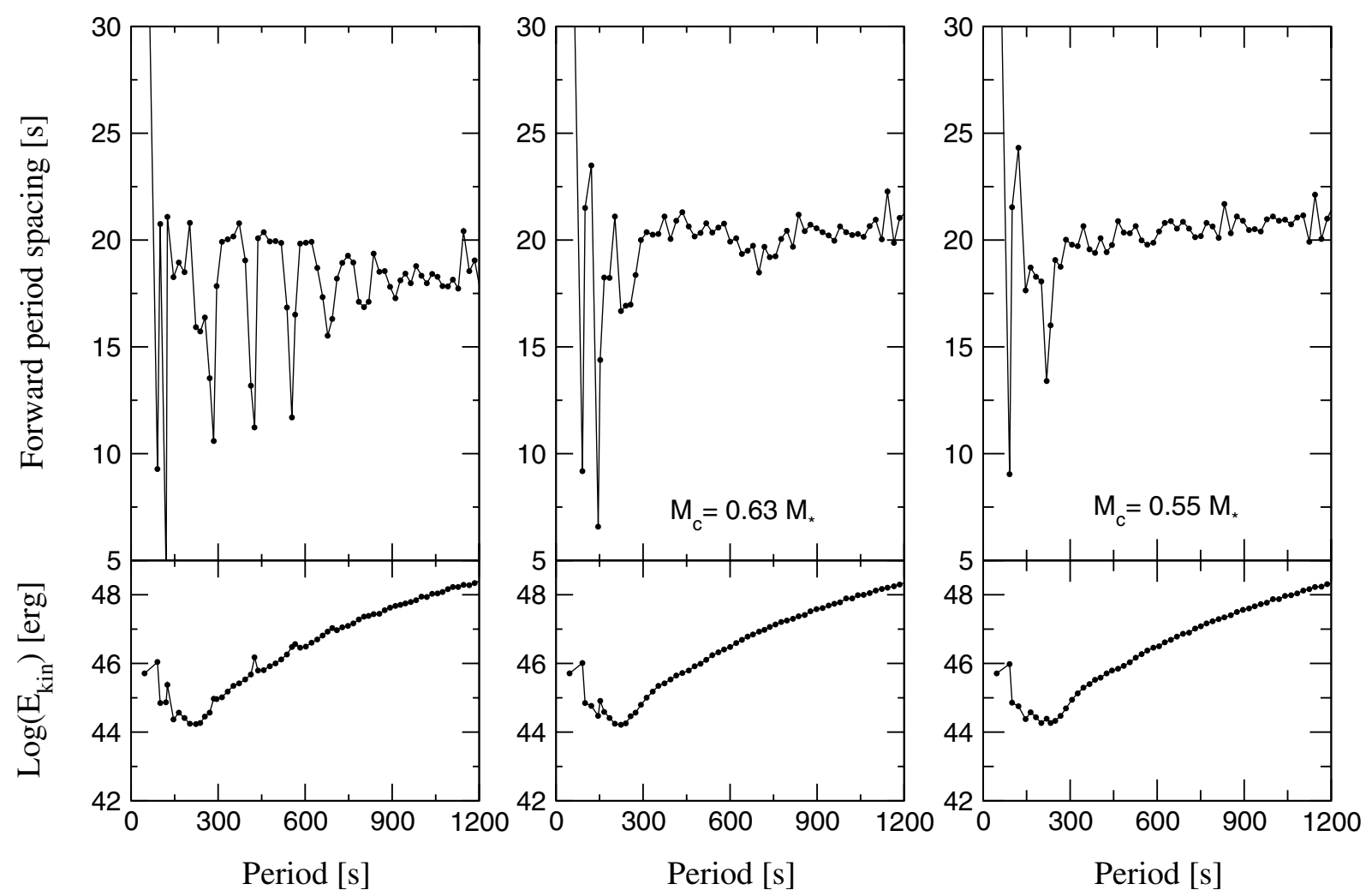

Fig. 9. The forward period spacing (upper panels) and the kinetic energy (lower panels) in terms of the periods of $\ell=2$ pulsation modes. The left panels correspond to a CO white dwarf model in which crystallization has not been taken into account. The central panels show the same quantities for a model at the same $T_{\text {eff }}$ in which crystallization has been considered but phase separation has not. Finally, the right panels show the case in which both crystallization and phase separation have been taken into account.

models are only computed for the sake of comparison and in order to exemplify the effects of crystallization in spite of the fact that they are not physically sound. The thick lines correspond to our fiducial models. That is, the thick solid line corresponds to an $\mathrm{ONe}$ model in which crystallization has been taken into account, whereas the thick dashed line corresponds to the $\mathrm{CO}$ white dwarf model in which both crystallization and phase separation were taken into account. The thin dashed line corresponds to a $\mathrm{CO}$ model in which crystallization but no phase separation was considered. The thin dotted line corresponds to a CO model in which neither crystallization nor phase separation were taken into account. Finally, the thin solid line indicates the results for an $\mathrm{ONe}$ model in which crystallization was completely disregarded. The results presented in Fig. 8 deserve several comments. Firstly, for all the cases considered $\Delta P_{\ell=2}$ is an increasing function of $T_{\text {eff }}$, as expected from the fact that the Brunt-Väisälä frequency decreases as the white dwarf cools down $-\chi_{\mathrm{T}}$ decreases as degeneracy increases, see Eq. (2). Secondly, it is noteworthy that if crystallization is neglected $\Delta P_{\ell=2}$ is smaller for the $\mathrm{ONe}$ models than for the $\mathrm{CO}$ ones. This trend is understood in terms of $N^{2}$ having noticeably larger values at the core region in the $\mathrm{ONe}$ models than in the $\mathrm{CO}$ models. Thus, the integral of Eq. (1) turns out to be larger for ONe models and, consequently, the asymptotic period spacing is smaller.

In contrast, for the cases in which crystallization has been considered, the values of $\Delta P_{\ell=2}$ of the ONe models are always larger than those of the $\mathrm{CO}$ ones. In part, this can be understood from the fact that the ONe model is characterized by a larger crystallized mass fraction than the $\mathrm{CO}$ one for a fixed $T_{\text {eff }}$. This in turn implies that the integral of $N / r$ in Eq. (1) is smaller and the resulting asymptotic period spacing in the ONe model is larger. With regard to the $\mathrm{CO}$ models, we note that when chemical rehomogenization is considered the asymptotic period spacing is slightly greater than in the situation in which rehomogenization is neglected. This can be understood on the basis that, when rehomogenization is not allowed to operate, the integral in Eq. (1) has an extra contribution from the overshoot-induced step via the strong peak at $\log \left(1-M_{\mathrm{r}} / M_{*}\right) \approx-0.7$ in the the Brunt-Väisälä frequency (see Fig. 1).

We now examine the forward period spacing, defined as $\Delta P_{k}=P_{k+1}-P_{k}$ ( $k$ being the radial overtone). This quantity, which is particularly sensitive to the details of the internal chemical structure of the white dwarf models, is usually employed to infer the mode trapping properties of ZZ Ceti stars. In addition, the theoretical period spacing can be directly compared with the observed one in a given star, whenever a sufficient number of consecutive periods (with the same harmonic degree $\ell$ ) can be measured. Here we restrict ourselves to present pulsational results corresponding to the same models analyzed in Figs. 5-7. We recall that these models are characterized by an effective temperature of $\approx 11800 \mathrm{~K}$, which is representative of ZZ Ceti stars. Figure 9 shows the $\ell=2$ period spacing distribution (top panels) and the kinetic energy of oscillation (bottom panels) as a function of the period for a $\mathrm{CO}$ 

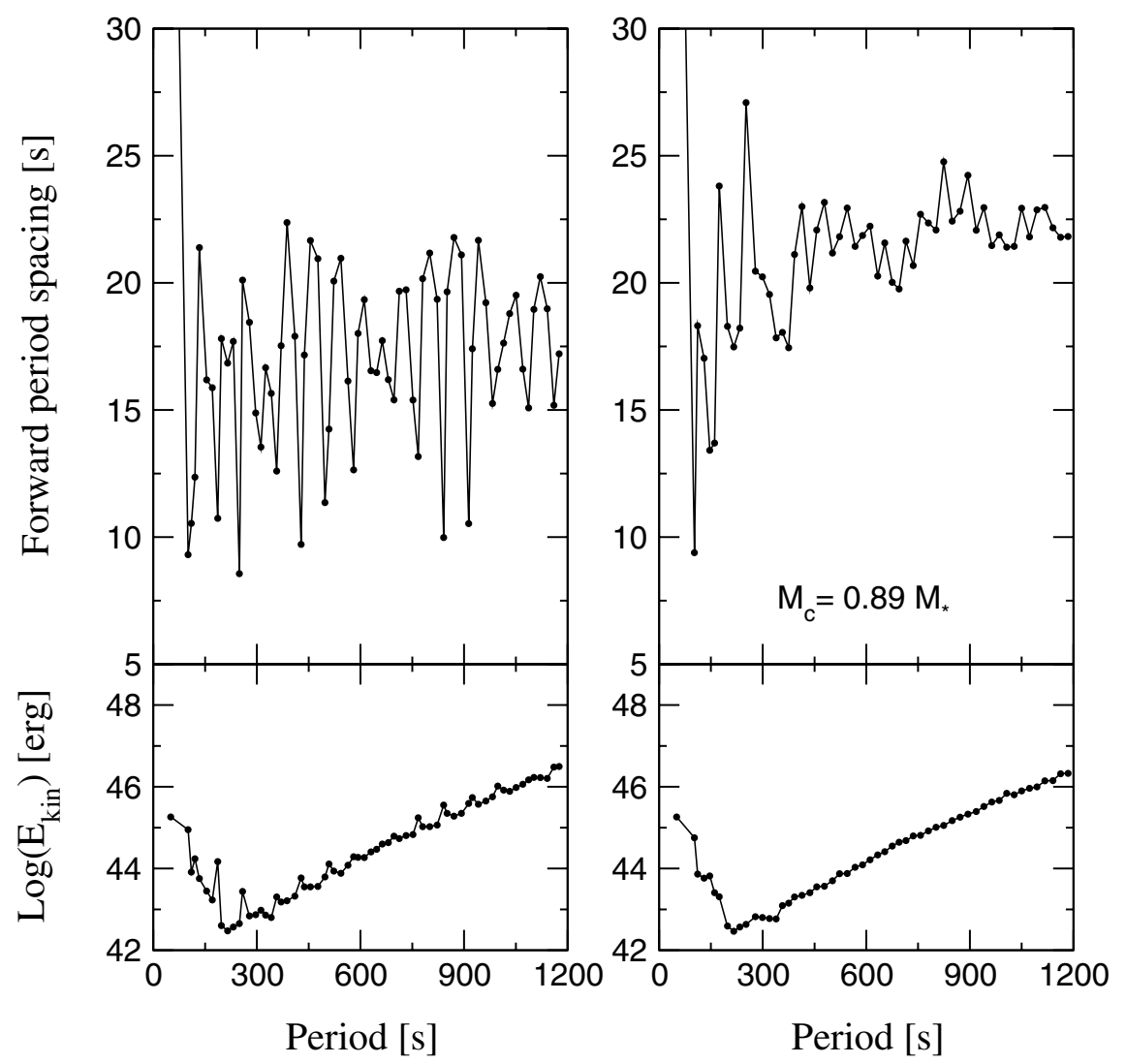

Fig. 10. Same as Fig. 9, but for the case of an ONe white dwarf.

white dwarf in which crystallization has been ignored (left panels), for a $\mathrm{CO}$ white dwarf in which crystallization was taken into account but no phase separation was considered (middle panels) and for the case in which both crystallization and phase separation were properly taken into account (right panels).

Clearly, when crystallization is considered the period spacing distribution is much less featured. Similar behavior has been reported by Montgomery \& Winget (1999) in calculations in which the crystallized mass fraction is considered as a free parameter. When crystallization is ignored, the internal boundary condition remains fixed at the center of the star. The resulting eigenspectrum is composed of some core-confined modes, some modes trapped in the outer layers, and the remainder normal modes (Córsico et al. 2002; Althaus et al. 2003). In this case the modes nearly preserve their character during the evolution. In contrast, when crystallization is considered (middle panels) the inner boundary condition moves together with the crystallization front, and the eigenfunctions of all modes are pulled out to the surface of the model. Under these circumstances, the resonance conditions determining which modes are trapped or confined are strongly modified as the degree of crystallization increases. This is clearly shown in the top middle panel of Fig. 9, which corresponds to a model in which the crystallization front is located at $M_{\mathrm{c}}=0.63 M_{*}$. Note that for the high- and intermediate-overtone modes the strong minima (associated with core-trapped modes) characterizing the $\Delta P_{k}$ distribution of the model for which crystallization was ignored (left panel), are not present in the case in which crystallization is considered (middle panel). A look at the lower panels of
Fig. 9 also shows that when crystallization is taken into account the differences between the kinetic energies of trapped, normal and confined modes are smaller. Another interesting point is that the mean period spacing increases when crystallization is considered, in agreement with the predictions of the asymptotic theory of pulsations and with the results of Montgomery \& Winget (1999).

We now examine the more realistic case in which phase separation is included in addition to crystallization (right panels). In view of the above considerations, we expect a smooth period spacing distribution. We note that the period spacing distribution (upper right panel) has a fairly clean structure for periods longer than $\approx 300 \mathrm{~s}$. In fact, the forward period spacing for modes with $k>12$ becomes very close to $20.6 \mathrm{~s}$, the value predicted by the asymptotic theory and given by Eq. (1). As already discussed, this is due mostly to the absence of any abrupt feature in the profile of the Brunt-Väisälä frequency. In fact, the model is characterized by a crystallized mass fraction of $\approx 0.55$, which is large enough for the ongoing chemical rehomogenization processes to erase the overshoot-induced step in the chemical profile (see Figs. 1 and 2).

As previously stated, the major aim of this work is to explore the possibility of using white dwarf asteroseismology to distinguish massive $\mathrm{CO}$ white dwarfs from those having $\mathrm{ONe}$ cores. In the right panels of Fig. 10 we show the distributions of $\Delta P_{k}$ and $E_{\text {kin }}$ that correspond to our fiducial ONe model at $T_{\text {eff }} \approx 11800 \mathrm{~K}$ (which corresponds to a crystallized mass $M_{\mathrm{c}} \approx 0.90 M_{*}$ ). For the sake of completeness in the left panels of this figure we also show the same distributions for the 
case in which crystallization was ignored. We note that, irrespective of the assumptions regarding crystallization for both types of models ( $\mathrm{CO}$ or $\mathrm{ONe})$, the period spacing diagrams of ONe models are noticeably different from those of the $\mathrm{CO}$ models. In particular, for the case in which crystallization was disregarded (left panels), the period spacing diagram of the ONe model shows strong and abundant features linked with mode trapping and confining, mostly driven by the structure at $-2 \lesssim \log \left(1-M_{\mathrm{r}} / M_{*}\right) \lessgtr-1$ in the Brunt-Väisälä frequency profile (see Fig. 7). When crystallization is taken into account, the period spacing distribution of the ONe model still has a complex structure but with a noticeably lower strength (right upper panel of Fig. 10). In particular, we note the presence of three minima at $\approx 300,600$ and $900 \mathrm{~s}$. However, the comparison of the period spacing diagrams in the right panels of Figs. 9 and 10 indicates that two models with the same stellar mass and effective temperature but differing in core composition (one having a core rich in carbon and oxygen, and the other mainly composed of oxygen and neon) should be characterized by a quite different pulsational spectrum.

An additional difference in the pulsational properties between the two types of models is found when the kinetic energies of the modes are compared. In fact, all the modes of the ONe model have lower energies than those of their counterparts of the $\mathrm{CO}$ model. Two facts help to clarify this issue. We recall that $E_{\text {kin }}$ is proportional to the density and to the squared radial and horizontal eigenfunctions. Firstly, the propagation regions in which the eigenmodes are allowed to oscillate have different sizes. Specifically, for the ONe white dwarf at $T_{\text {eff }} \approx 11800 \mathrm{~K}$, we find that $90 \%$ of the stellar mass has already crystallized. Hence, in this high-density region nonradial $g$-modes are excluded. Therefore, the modes are able to propagate only in the remaining external, low-density region. In the case of the CO model at the same $T_{\text {eff }}$, the crystallized mass fraction is aproximately 0.55 , and so the modes can propagate in regions characterized by considerably quite high densities. Secondly, we have found that for the case of ONe models the amplitudes of the eigenfunctions are considerably smaller than those of their counterparts in the $\mathrm{CO}$ models. As a final remark we note that the mean period spacing of the pulsation modes corresponding to ONe white dwarfs is slightly larger than that of the CO models. This is again in agreement with the predictions of the asymptotic theory, as shown in Fig. 8.

In closing, we stress that the main differences in the pulsational spectrum between $\mathrm{ONe}$ and $\mathrm{CO}$ models are due to the presence of a strong step in the core chemical profile of the ONe model. As mentioned, this step marks the outer edge of the mixing zone induced by the negative molecular weight of the $\mathrm{ONe}$ white dwarf progenitor. However, additional mixing episodes beyond this external border may wipe out the chemical step. In that case we would expect the period pattern to be modified to a considerable extent. This is indeed borne out by Fig. 11, in which the period spacing is computed under the assumption that $B=0$ in the region at $-2 \lesssim \log \left(1-M_{\mathrm{r}} / M_{*}\right) \lessgtr-1$ and the resulting run of the Brunt-Väisälä frequency is that displayed by a dashed line in Fig. 7. We are aware that this procedure does not eliminate completely the effects of the strong chemical feature on the period spectrum, because the

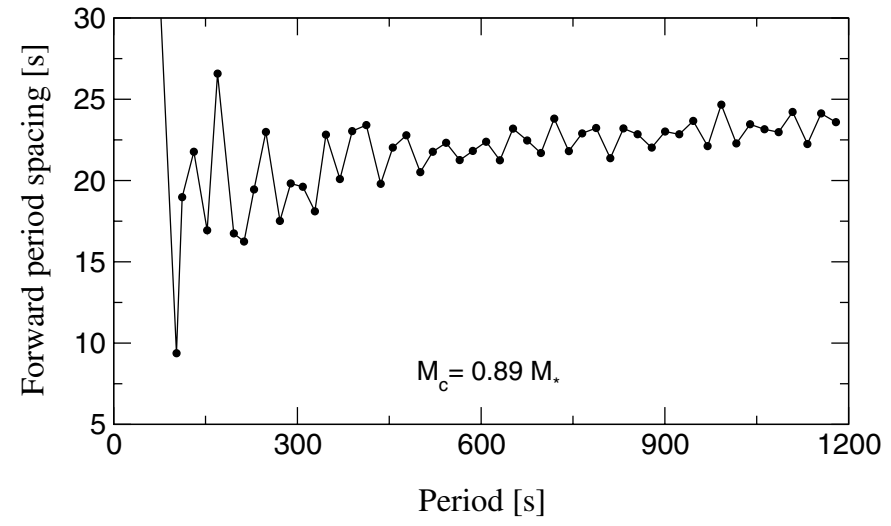

Fig. 11. Same as the upper-right panel of Fig. 10, but for the case in which the effect of the strong step in the chemical profile at $-2 \lesssim$ $\log \left(1-M_{\mathrm{r}} / M_{*}\right) \lessgtr-1$ (see Fig. 4) on the shape of the Brunt-Väisälä frequency has been neglected by setting $B=0$ in that region (see Fig. 7).

change in density associated with this chemical interface is still present in the computation of $N$ (see Eq. (2)). However, we consider this approximation reliable enough for our purposes here. Note that the forward period spacing becomes much less pronounced than when the Brunt-Väisälä frequency is computed self-consistently (right panel of Fig. 10), and instead, it is reminiscent to the results obtained for the $\mathrm{CO}$ model (see right panel of Fig. 9). This aspect would render the current asteroseismological techniques much less able to yield the actual core composition of the massive white dwarfs. We note however, that the average period spacing remains virtually unchanged even in the presence of such extra mixing episode.

\section{Conclusions}

In this work we have computed the evolution of massive white dwarfs with hydrogen-rich envelopes and carbon/oxygen and oxygen/neon cores with the major aim of comparing their adiabatic pulsational properties. Particular attention was given to the formation of the core chemical profile during the pre-white dwarf evolutionary stages and to the evolution of the chemical abundance distribution caused by element diffusion during the white dwarf regime. In addition, the chemical rehomogenization induced by phase separation upon crystallization as well as the effects of crystallization on the oscillation eigenmodes have been fully taken into account.

Our results show that because the chemical interfaces in the envelope are markedly smoothed by diffusion, the chemical structure of the core mostly determines the structure of the period spectrum. This result reinforces the conclusions arrived at in Córsico et al. $(2001,2002)$ and Althaus et al. (2003) about the role of time-dependent element diffusion in pulsatng calculations. Moreover, we find that, in contrast with their carbon/oxygen core counterparts, oxygen/neon white dwarfs are characterized by strong non-uniformities in their period spacing distribution. The lack of a featured period spacing distribution characterizing white dwarfs with carbon/oxygen cores arises from the fact that the mixing episode induced by phase separation as crystallization proceeds completely wipes out any 
feature in the shape of the innermost chemical profile built up during the evolutionary stages prior to the white dwarf formation. In addition, we found that the average period spacing characterizing our oxygen/neon models is appreciably larger than that of the carbon/oxygen counterparts having the same stellar mass and effective temperature. This is true even in the presence of extra mixing episodes that could eventually remove the sharp abundance discontinuity left in the oxygen/neon models. Finally, we also find quite different kinetic energy spectra for the two types of models.

Hence, it turns out that the asteroseismological techniques usually employed in variable white dwarf studies constitute, in principle, a powerful tool for distinguishing massive carbon/oxygen white dwarfs from those having oxygen/neon cores. In this regard, the most massive ZZ Ceti star presently known, BPM 37093, is of particular interest since the value of its stellar mass of $\sim 1.05 M_{\odot}$ places it close to the theoretical lower limit for an oxygen/neon white dwarf to be formed. Nonetheless, the scarcity of detected periods (eight periods) in the light curve of BPM 37093 renders conclusive asteroseismological inferences in this case very difficult. However, since the average period spacing is larger for the oxygen/neon ones, a comparison with the observed value would help to get a clear insight into the actual core composition of this massive white dwarf. We feel that this aspect would deserve to be explored in the frame of a set of white dwarf models with various stellar masses. Ongoing attempts to constrain the fundamental properties of BPM 37093 using asteroseismological data should allow us to shed new light on the scenarios leading to the formation of single massive white dwarfs and on the nature of their progenitors.

Acknowledgements. This work was partially supported by the Instituto de Astrofísica La Plata, by the MCYT grants AYA04094C03-01 and 02, by the European Union Feder funds, and by the CIRIT. L.G.A. also acknowledges the Spanish MCYT for a Ramón y Cajal Fellowship.

\section{References}

Alexander, D. R., \& Ferguson, J. W. 1994, ApJ, 437, 879

Althaus, L. G., Serenelli, A. M., \& Benvenuto, O. G. 2001a, ApJ, 554, 1110

Althaus, L. G., Serenelli, A. M., \& Benvenuto, O. G. 2001b, MNRAS, 323,471

Althaus, L. G., Serenelli, A. M., Córsico, A. H., \& Benvenuto, O. G. 2002, MNRAS, 330, 685

Althaus, L. G., Serenelli, A. M., Córsico, A. H., \& Montgomery, M. H. 2003, A\&A, 404, 593

Angulo, C. Arnould, M., Rayet, M., et al. 1999, Nucl. Phys. A, 656, 3

Barstow, M. A., Jordan, S., O’Donoghue, D., et al. 1995, MNRAS, 277, 991

Bradley, P. A. 1998, ApJS, 116, 307

Bradley, P. A. 2001, ApJ, 552, 326
Brassard, P., Fontaine, G., Wesemael, F., \& Hansen, C. J. 1992, ApJS, 80, 369

Brassard, P., Fontaine, G., Wesemael, F., Kawaler, S. D., \& Tassoul, M. 1991, ApJ, 367, 601

Burgers, J. M. 1969, Flow Equations for Composite Gases (New York: Academic Press)

Caughlan, G. R., \& Fowler, W. A. 1988, Atom. Data \& Nucl. Data Tables, 40, 290

Córsico, A. H. 2003, Ph.D., University of La Plata (http://www. fcaglp.unlp.edu.ar/evolgroup/)

Córsico, A. H., Althaus, L. G., Benvenuto, O. G., \& Serenelli, A. M. 2001, A\&A, 380, L17

Córsico, A. H., Althaus, L. G., Benvenuto, O. G., \& Serenelli, A. M. 2002, A\&A, 387, 531

Dahn, C. C., Bergeron, P., Liebert, J., et al. 2004, ApJ, 605, 400

D’Antona, F., \& Mazzitelli, I. 1996, ApJ, 470, 1093

Dupuis, J., Chayer, P., Vennes, S., Allard, N. F., \& Hébrard, G. 2003, ApJ, 598, 486

Ferrario, L., Vennes, S., Wickramasinghe, D. T., Bailey, J. A., \& Christian, D. J. 1997, MNRAS, 292, 205

García-Berro, E., \& Iben, I. 1994, ApJ, 434, 306

García-Berro, E., Ritossa, C., \& Iben, I. 1997, ApJ, 485, 765

García-Berro, E., Hernanz, M., Mochkovitch, R., \& Isern, J. 1988, A\&A, 193, 141

Gil-Pons, P., García-Berro, E., José, J., Hernanz, M., \& Truran, J. W. 2003, A\&A, 407, 1021

Grossman, S. A., \& Taam, R. E. 1996, MNRAS, 283, 1165

Guerrero, J., García-Berro, E., \& Isern, J. 2004, A\&A, 413, 257

Herwig, F. 2000, A\&A, 360, 952

Iben, I., Ritossa, C., \& García-Berro, E. 1997, ApJ, 489, 772

Iglesias, C. A., Rogers, \& F. J. 1996, ApJ, 464, 943

Kanaan, A., Kepler, S. O., Giovannini, O., \& Diaz, M. 1992, ApJ, 390, L89

Kawaler, S. D., Winget, D. E., \& Hansen, C. J. 1985, ApJ, 295, 547

Magni, G., \& Mazzitelli, I. 1979, A\&A, 72, 134

Montgomery, M. H., \& Winget, D. E. 1999, ApJ, 526, 976

Montgomery, M. H., Klumpe, E. W., Winget, D. E., \& Wood, M. A. 1999, ApJ, 525, 482

Osaki, Y., \& Hansen, C. J. 1973, ApJ, 185, 277

Pfeiffer, B., Vauclair, G., Dolez, N., et al. 1996, A\&A, 314, 182

Ritossa, C., García-Berro, E., \& Iben, I. 1996, ApJ, 460, 489

Salaris, M., Domínguez, I., García-Berro, E., et al. 1997, ApJ, 486, 413

Schmidt, G. D., Bergeron, P., Liebert, J., \& Saffer, R. A. 1992, ApJ, 394, 603

Segretain, L. 1996, A\&A, 310, 485

Segretain, L., \& Chabrier, G. 1993, A\&A, 271, L13

Segretain, L., Chabrier, G., Hernanz, M., et al. 1994, ApJ, 434, 641

Segretain, L., Chabier, G., \& Mochkovitch, R. 1997, ApJ, 481, 355

Straniero, O., Domínguez, I., Imbriani, G., \& Piersanti, L. 2003, ApJ, 583, 878

Tassoul, M., Fontaine, G., \& Winget, D. E. 1990, ApJS, 72, 335

Winget, D. E., Kepler, S. O., Kanaan, A., Montgomery, M. H., \& Giovannini, O. 1997, ApJ, 487, L191 